\title{
Parikh Matrices: Subword Indicators and Degrees of Ambiguity
}

\author{
Arto Salomaa ${ }^{(\otimes)}$ \\ Turku Centre for Computer Science, University of Turku, Quantum Building 392, \\ 20014 Turun yliopisto, Finland \\ asalomaa@utu.fi
}

\begin{abstract}
The quantity $|w|_{u}$, the number of occurrences of a word $u$ as a (scattered) subword of a word $w$ gives important numerical information about the word $w$. Properly chosen values $|w|_{u}$, for different $u$ 's, characterize the word $w$ completely. Certain upper triangular matrices, customarily referred to as Parikh matrices have turned out to be very useful for computing numbers $|w|_{u}$. This partially expository paper discusses some highlights and open problems of the theory of Parikh matrices and subword occurrences. Special emphasis is on subword indicators and degrees of ambiguity.
\end{abstract}

\section{Introduction}

The most direct numerical fact about a word $w$ is its length, in symbols $|w|$. The Parikh vector, [6], $\Psi(w)=\left(i_{1}, \ldots, i_{n}\right)$ indicates the number of occurrences of the letter $a_{j}, 1 \leq j \leq n$, in $w$, provided $w$ is over the alphabet $\left\{a_{1}, \ldots, a_{n}\right\}$. To get more information about a word, one has to focus the attention to the number of occurrences of a specific subword in the given word. The word $u$ being a subword of $w$ means that $w$, as a sequence of letters, contains $u$ as a subsequence. More specifically, there exist letters $x_{1}, \ldots, x_{k}$ and words $y_{0}, \ldots, y_{k}$, some of them possibly empty, such that

$$
u=x_{1} \ldots x_{k} \quad \text { and } \quad w=y_{0} x_{1} y_{1} \ldots x_{k} y_{k} .
$$

We also consider factors $u$ of a word $w: u$ is a factor of $w$ if there are words $x$ and $y$ such that $w=x u y$. Throughout this paper, we understand subwords and factors in the way mentioned. (In classical language theory our subwords are usually called "scattered subwords", whereas our factors are called "subwords".)

We use the notation $|w|_{u}$ for the number of occurrences of the word $u$ as a subword of the word $w$. This number can be defined also as follows. Occurrences can be viewed as vectors. If $|u|=t$, each occurrence of $u$ in $w$ can be identified as the $t$-tuple $\left(i_{1}, \ldots, i_{t}\right)$ of increasing positive integers, where for $1 \leq j \leq t$, the $j$ th letter of $u$ is the $i_{j}$ th letter of $w$. Then $|w|_{u}$ equals the number of such vectors. For instance, the 3 occurrences of $u=a b$ in $w=a b a b a$ are $(1,2),(1,4)$ and $(3,4)$. We also make the convention that, for any $w$ and the empty word $\lambda$, $|w|_{\lambda}=1$. 
In [2] the number $|w|_{u}$ is denoted as a "binomial coefficient" $|w|_{u}=\left(\begin{array}{l}w \\ u\end{array}\right)$. If $w$ and $u$ are words over a one-letter alphabet, $w=a^{i}, u=a^{j}$, then $|w|_{u}$ equals the ordinary binomial coefficient: $|w|_{u}=\left(\begin{array}{l}i \\ j\end{array}\right)$. Our convention concerning the empty word reduces to the fact that $\left(\begin{array}{l}i \\ 0\end{array}\right)=1$. However, the notation using binomial coefficients is not convenient in case of general alphabets.

Assume that $\Sigma$ is an alphabet containing the letters $a$ and $b$. Then, for any word $w$,

$$
\left(|w|_{a}\right) \cdot\left(|w|_{b}\right)=|w|_{a b}+|w|_{b a} .
$$

This simple equation is one of the few general facts about occurrences of subwords. A slight variation immediately leads to difficulties. No explicit characterization is known, [4], for the relation between $\left(|w|_{u},|w|_{v}\right)$ and $\left(|w|_{u v},|w|_{v u}\right)$, where $u, v, w$ are arbitrary words.

\section{Parikh Matrices and Generalizations}

We now consider a sharpening of the Parikh mapping, introduced in [3], which gives information also about the location of different letters in a word. The mapping uses upper triangular square matrices, with non-negative integer entries, 1's on the main diagonal and 0's below it. The set of all such triangular matrices is denoted by $\mathcal{M}$, and the subset of all matrices of dimension $k \geq 1$ is denoted by $\mathcal{M}_{k}$. Two words with the same Parikh matrix always have the same Parikh vector, but two words with the same Parikh vector have in general different Parikh matrices.

The notion of a Parikh matrix mapping is defined as follows. In what follows, small letters $a, b, c, d$, possibly with indices, from the beginning of the English alphabet denote letters of the formal alphabet.

Definition 1. Let $\Sigma=\left\{a_{1}, \ldots, a_{k}\right\}$ be an ordered alphabet. The Parikh matrix mapping, denoted $\Psi_{k}$, is the morphism $\Psi_{k}: \Sigma^{*} \rightarrow \mathcal{M}_{k+1}$, defined as follows. Consider an integer $q, 1 \leq q \leq k$. If we denote $\Psi_{k}\left(a_{q}\right)=\left(m_{i, j}\right)_{1 \leq i, j \leq(k+1)}$, then, for each $1 \leq i \leq(k+1), m_{i, i}=1, m_{q, q+1}=1$, all other elements of the matrix $\Psi_{k}\left(a_{q}\right)$ being 0 .

The following theorem, describes the basic properties of the Parikh matrix mapping. The proof is an easy induction on the length of the word $w$. Details can be found in [3]. For $\Sigma=\left\{a_{1}, \ldots, a_{k}\right\}$, we denote by $a_{i, j}$ the word $a_{i} a_{i+1} \ldots a_{j}$, where $1 \leq i \leq j \leq k$.

Theorem 1. Consider $\Sigma=\left\{a_{1}, \ldots, a_{k}\right\}$ and $w \in \Sigma^{*}$. The matrix $\Psi_{k}(w)=$ $\left(m_{i, j}\right)_{1 \leq i, j \leq(k+1)}$, has the following properties:

- $m_{i, j}=0$, for all $1 \leq j<i \leq(k+1)$,

- $m_{i, i}=1$, for all $1 \leq i \leq(k+1)$,

- $m_{i, j+1}=|w|_{a_{i, j}}$, for all $1 \leq i \leq j \leq k$. 
Parikh matrices give much more information about a word $w$ than Parikh vectors. Theorem 1 tells that the second diagonal of the Parikh matrix of $w$ gives the Parikh vector of $w$. The next diagonals give information about the order of letters in $w$.

There is an extensive literature concerning Parikh matrices. The reader is referred to $[1,5,7-10,13-19]$ for various aspects of the theory, many of which will be discussed below. Our emphasis lies in the core of the theory. We do not discuss related issues such as subword histories, [4], or position indices, [11]. Some new notions are introduced in the two last sections of the paper.

The Parikh matrix mapping yields the numbers $|w|_{u}$, where $u$ is a factor of the word $a_{1} \ldots a_{k}$. Knowledge about numbers such as $|w|_{\text {baa }}$ will not be obtained. This drawback can be corrected by considering generalized Parikh matrix mappings, originally introduced in $[13,14]$.

The generalized Parikh matrix mapping $\Psi_{v}$ tells the number of occurrences (as a subword) of factors of an arbitrary word $v=c_{1} \cdots c_{t}$, where each $c$ is a letter. Repetitions of letters are possible.

Definition 2. Consider a word $v=c_{1} \cdots c_{t}$ of length $t$ over the alphabet $\Sigma$. The generalized Parikh matrix mapping, denoted $\Psi_{v}$, is the morphism: $\Psi_{v}: \Sigma^{*} \rightarrow \mathcal{M}_{t+1}$, defined as follows. Assume that the letter a occurs in the word $v$ in positions $j_{1}, \ldots, j_{r}$. Denote $\Psi_{v}(a)=\left(n_{i, j}\right)_{1 \leq i, j \leq(t+1)}$. Then for each $1 \leq i \leq(t+1), n_{i, i}=1$. Moreover, $n_{i, i+1}=1$ if $i$ is one of the numbers $j_{1}, \ldots, j_{r}$. All other elements of the matrix $\Psi_{v}(a)$ are 0 .

A result analogous to Theorem 1 is that the entry $n_{i, j+1}, 1 \leq i \leq j \leq t$, in the matrix corresponding to a word $w$ equals the number $|w|_{c_{i} \cdots c_{j}}$. The proof of this result, based on using matrix products, is again straightforward.

For instance, let $v=a b a b a$ and let $w$ be arbitrary. Then

$$
\Psi_{a b a b a}(w)=\left(\begin{array}{ccclll}
1 & |w|_{a}|w|_{a b} & |w|_{a b a} & |w|_{\text {abab }} & |w|_{\text {ababa }} \\
0 & 1 & |w|_{b} & |w|_{b a} & |w|_{b a b} & |w|_{b a b a} \\
0 & 0 & 1 & |w|_{a} & |w|_{a b} & |w|_{a b a} \\
0 & 0 & 0 & 1 & |w|_{b} & |w|_{b a} \\
0 & 0 & 0 & 0 & 1 & |w|_{a} \\
0 & 0 & 0 & 0 & 0 & 1
\end{array}\right) .
$$

Choosing $w=$ baabaabaab, we obtain

$$
\Psi_{a b a b a}(\text { baabaabaab })=\left(\begin{array}{cccccc}
1 & 6 & 12 & 12 & 16 & 8 \\
0 & 1 & 4 & 12 & 20 & 16 \\
0 & 0 & 1 & 6 & 12 & 12 \\
0 & 0 & 0 & 1 & 4 & 12 \\
0 & 0 & 0 & 0 & 1 & 6 \\
0 & 0 & 0 & 0 & 0 & 1
\end{array}\right) .
$$

The following fundamental result, [13], shows how every generalized Parikh matrix is related to a Parikh matrix. The result can be used for transferring facts concerning subword occurrences based on Parikh matrices to corresponding facts 
based on generalized Parikh matrices. A typical example of this is the inequality considered in [12].

Theorem 2. For every generalized Parikh matrix mapping $\Psi_{v}(w),|v|=t$, an ordered alphabet $\Sigma_{t}=\left\{a_{1}, \ldots, a_{t}\right\}$ and a word $w^{\prime} \in \Sigma_{t}^{*}$ such that

$$
\Psi_{v}(w)=\Psi_{t}\left(w^{\prime}\right)
$$

can be effectively constructed. Here, $\Psi_{t}$ is a Parikh matrix mapping.

Proof. Number the occurrences of the letters in $v$ by $1, \ldots, t$. The numbers $1, \ldots, t$ constitute the alphabet $\Sigma_{t}$. Consider the alphabet $\Sigma$ of $v$. A morphism $h$ of $\Sigma^{*}$ into $\Sigma_{t}^{*}$ is defined as follows. Let $a \in \Sigma$ appear in $v$ in positions

$$
p_{1}, p_{2}, \ldots, p_{q}, 1 \leq q \leq t, p_{i}<p_{i+1}, 1 \leq i \leq q-1 .
$$

Then we define

$$
h(a)=p_{q} p_{q-1} \cdots p_{1} .
$$

Finally, for $w \in \Sigma^{*}$, we choose $w^{\prime}=h(w)$.

In our example $v=$ ababa above, $h(a)=531$ and $h(b)=42$. Hence, for $w=$ baabaabaab, we obtain

$$
w^{\prime}=42531531425315314253153142 .
$$

For the (ordered) alphabet $\Sigma_{5}=\{1,2,3,4,5\}$, we now have

$$
\Psi_{5}(42531531425315314253153142)=\left(\begin{array}{llllll}
1 & 6 & 12 & 12 & 16 & 8 \\
0 & 1 & 4 & 12 & 20 & 16 \\
0 & 0 & 1 & 6 & 12 & 12 \\
0 & 0 & 0 & 1 & 4 & 12 \\
0 & 0 & 0 & 0 & 1 & 6 \\
0 & 0 & 0 & 0 & 0 & 1
\end{array}\right) .
$$

The result equals the generalized Parikh matrix obtained above.

The reverse order of the numbers $p_{i}$ in the definition of the morphism is necessary because, otherwise, additional false occurrences are created. For instance, choose $v=a a$ and define $h(a)=12$. Then $\Psi_{v}(a a a) \in \mathcal{M}_{3}$ has the number 3 in the upper right-hand corner. However, $\Psi_{2}(121212)$ has the number 6 in the upper right-hand corner.

The proof of Theorem 2 is now completed by induction on the length of the word $w$. Consider the morphism $h$ and the word $w^{\prime}$ defined above. Clearly the theorem holds if $w$ is a letter. Assuming that Theorem 2 holds for words $w$ of length $n$ and consider $h(a) \in \Sigma_{t}^{+}, a \in \Sigma$. Now we obtain $\Psi_{v}(w a)=\Psi_{v}(w) \Psi_{v}(a)$. By the induction hypothesis, $\Psi_{v}(w)=\Psi_{t}(h(w))$. Hence,

$$
\Psi_{v}(w a)=\Psi_{t}(h(w)) \Psi_{v}(a)=\Psi_{t}(h(w)) \Psi_{t}(h(a)) .
$$

The equation

$$
\Psi_{v}(w a)=\Psi_{t}(h(w a))
$$

follows by matrix multiplication. The factor $\Psi_{t}(h(a))$ increases appropriate entries in the matrix $\Psi_{t}(h(w))$. 
Coming back to our example, assume that we have established that

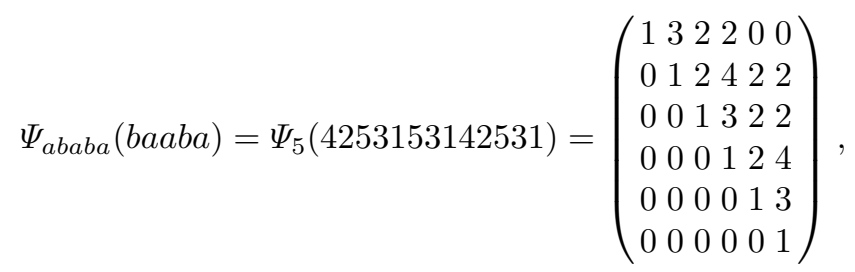

and want to establish

$$
\Psi_{a b a b a}(\text { baabaa })=\Psi_{5}(4253153142531531) .
$$

Clearly,

$$
\Psi_{5}(531)=\left(\begin{array}{llllll}
1 & 1 & 0 & 0 & 0 & 0 \\
0 & 1 & 0 & 0 & 0 & 0 \\
0 & 0 & 1 & 1 & 0 & 0 \\
0 & 0 & 0 & 1 & 0 & 0 \\
0 & 0 & 0 & 0 & 1 & 1 \\
0 & 0 & 0 & 0 & 0 & 1
\end{array}\right)
$$

Hence,

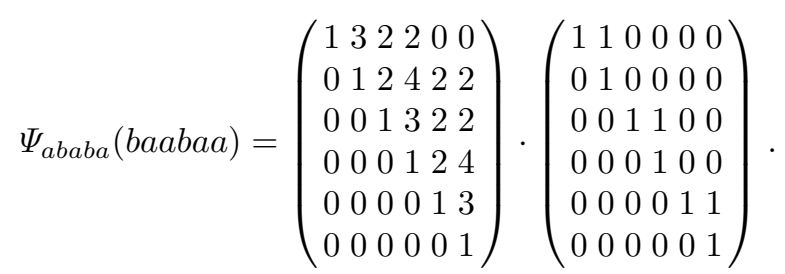

Finally, we obtain

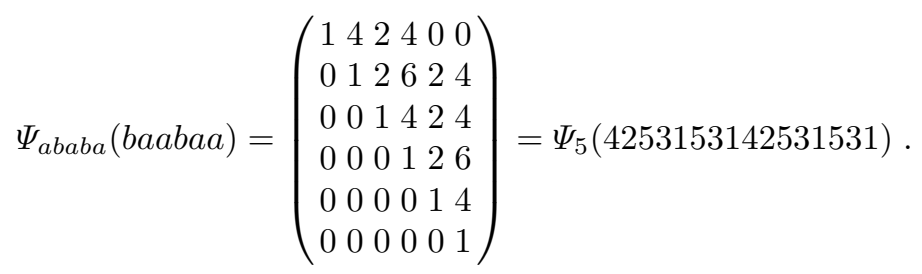

\section{Ambiguity and Decision Problems}

Only in some special cases the matrix $\Psi(w)$ or the generalized matrix $\Psi_{v}(w)$ defines uniquely the word $w$. We begin with the following definition. (We skip the corresponding definition for generalized Parikh matrices.)

Definition 3. Let $k$ and $\Psi_{k}$ be as in Definition 1. Two words $w_{1}, w_{2} \in \Sigma_{k}^{*}$ are termed $M$-equivalent, in symbols $w_{1} \equiv_{M} w_{2}$, if $\Psi_{k}\left(w_{1}\right)=\Psi_{k}\left(w_{2}\right)$. A word $w \in \Sigma_{k}^{*}$ is termed $M$-unambiguous if there is no word $w^{\prime} \neq w$ such that $w \equiv_{M} w^{\prime}$. Otherwise, $w$ is termed $M$-ambiguous. If $w \in \Sigma_{k}^{*}$ is $M$-unambiguous (resp. $M$-ambiguous), then also the Parikh matrix $\Psi_{k}(w)$ is called unambiguous (resp. ambiguous). 
A word being $M$-unambiguous means that it is uniquely determined by its Parikh matrix. If the analogous notion is defined for Parikh vectors, then only the words over a one-letter alphabet are Parikh vector unambiguous.

In the sequel we will often drop the letter $M$ and speak simply of unambiguous and ambiguous words. It should be emphasized that in all notions involving Parikh matrices we deal with a specific ordering of the basic alphabet $\Sigma_{k}$.

Obviously, unambiguous words cannot possess ambiguous factors. More specifically, if a word $y \in \Sigma_{k}^{*}$ is ambiguous, so is every word $x y z$, where $x, z \in \Sigma_{k}^{*}$. However, unambiguous words may possess ambiguous subwords. For instance, the unambiguous word $a b c b a$ has the ambiguous subword $a b b a$.

Some short ambiguous words are listed in the following lemma. The proof is easy and is omitted.

Lemma 1. Consider the alphabet $\Sigma_{k}=\left\{a_{1}, \ldots, a_{k}\right\}$. The words

$$
a_{i} a_{i+j} \text { and } a_{i+j} a_{i}, 1 \leq i \leq k-2,2 \leq j \leq k-i
$$

are ambiguous. So are each of the words

$$
\begin{array}{ll}
a_{i} a_{i+1} a_{i+1} a_{i}, & a_{i+1} a_{i} a_{i} a_{i+1}, \\
a_{i} a_{i+1} a_{i}^{j} a_{i+1} a_{i}, & a_{i+1} a_{i}^{j+2} a_{i+1}, \\
a_{i+1} a_{i} a_{i+1}^{j} a_{i} a_{i+1}, & a_{i} a_{i+1}^{j+2} a_{i},
\end{array}
$$

where $1 \leq i \leq k-1$ and $j \geq 0$.

Ambiguous words can be characterized explicitly in case of a two-letter alphabet. For a proof of the following result the reader is referred to, for instance, [7]. An explicit characterization of unambiguous words over a three-letter alphabet was given in [14].

Theorem 3. A word in $\{a, b\}^{*}$ is ambiguous if and only if it contains factors $a b$ and ba which do not overlap. A word is unambiguous if and only if it belongs to the language denoted by the regular expression

$$
a^{*} b^{*}+b^{*} a^{*}+a^{*} b a^{*}+b^{*} a b^{*}+a^{*} b a b^{*}+b^{*} a b a^{*} .
$$

The regular language mentioned in Theorem 3 is accepted by a five-state non-deterministic finite automaton.

A notion important in connection with $M$-equivalence is the notion of a print introduced and investigated in $[14,15]$.

Definition 4. A word $w^{\prime}$ is the print of $w$ if $w^{\prime}$ results from $w$ by replacing every factor $a^{i}, i>1$, where $a$ is a letter, with a. A word is printed if it equals its print.

For instance, the print of $a c^{4} a^{2} c^{2} a b^{8} a b c^{3} b^{5}$ is $a c a c a b a b c b$.

It was shown in [15] that, for each alphabet, the set of unambiguous printed words is finite. An upper bound for the size of this set, depending on the size of the alphabet, can be effectively computed.

The following related theorem is established in also [15]. 
Theorem 4. Consider the alphabet $\Sigma_{k}=\left\{a_{1}, \ldots, a_{k}\right\}, k \geq 3$. There is an integer $N(k)$ such that every factor of length $\geq N(k)$ of the infinite word $\left(a_{1} a_{2} a_{3} \cdots a_{k}\right)^{\omega}$ is ambiguous.

The following Şerbănuţă's conjecture was open for a long time.

Conjecture 1. The unambiguity of a word implies the unambiguity of its print.

The conjecture holds for alphabets with 2 or 3 letters. It has been shown false in [19] for bigger alphabets. Indeed, the printed word cbcbabcdcbabcbc is ambiguous because it is $M$-equivalent with the word bccabcbdbcbaccb. However, the word $c b c b a b c^{2} d c b a b c b c$ is unambiguous.

In view of the result concerning unambiguous prints, Conjecture 1 would imply that all unambiguous words are found among those having "short" prints. Thus, all words with prints whose length exceeds a certain bound are ambiguous. This might still be true, since the counterexample to Conjecture 1 concerns a word with a short print. We would like to present the following conjecture.

Conjecture 2. There is a constant $k$, depending on the cardinality of the alphabet, such that, if the length of the print of a word $w$ exceeds $k$, then word $w$ is ambiguous.

Typical decision problems concerning Parikh matrices are the following ones.

1. Is a given matrix a Parikh matrix?

2. Given a word, decide whether or not it is $M$-ambiguous.

3. Are two given words $M$-equivalent?

The problem (3) amounts to computing two Parikh matrices and is clearly of polynomial time complexity. Straightforward algorithms for the problems (1) and (2), where one tests through all of the finitely many possibilities, result in an exponential time complexity. However, a really explicit characterization of Parikh matrices and unambiguous words is still missing.

It is easy to give examples of different Parikh matrices whose second and third diagonals coincide. However, it is not so easy to give examples of different Parikh matrices whose second, third and fourth diagonals coincide. Indeed, it was sometimes conjectured that such examples do not exist. It turns out that, no matter how many diagonals we fix, the Parikh matrix is still not necessarily determined.

Let $\Sigma_{k}, k \geq 2$, and $\Psi_{k}$ be as in Definition 1 . Consider integers $i$ and $j$, $1 \leq i<j \leq k+1$. The entry $(i, j)$ is said to be independent in the mapping $\Psi_{k}$ if there are words $w, w^{\prime} \in \Sigma_{k}^{*}$ such that the matrices $\Psi_{k}(w)$ and $\Psi_{k}\left(w^{\prime}\right)$ coincide elsewhere, but the $(i, j)$ th entries in the two matrices are different. The following result was established in [9].

Theorem 5. Every entry is independent in the mapping $\Psi_{k}$. 


\section{Subword Indicators}

In the original definition of a Parikh matrix mapping, Definition 1, we considered the ordered alphabet $\Sigma_{k}=\left\{a_{1}, \ldots, a_{k}\right\}$. The resulting matrices provided information about the subword occurrences (in the argument word of the mapping) of all factors of the word $a_{1} \cdots a_{k}$. Similar information about the subword occurrences of all factors of an arbitrary word $v$ was obtained in the matrices of a generalized Parikh matrix mapping. Going back to Definition 2, we refer to the word $v$ as the subword indicator of the generalized matrix mapping.

Thus, the word $a_{1} \cdots a_{k}$ is the subword indicator of the mapping in Definition 1. An extensive problem area, not at all investigated so far, consists of studying the change in the matrices affected by a change of the subword indicator. A special case is to study cases, where a change in the subword indicator does not affect the matrix, for some specific argument words. The following result gives a simple example.

Lemma 2. The equation

$$
\Psi_{a b}(w)=\Psi_{b a}(w), w \in\{a, b\}^{*}
$$

holds only for ambiguous words $w$.

Proof. Considering the entries in the matrices, we infer that a necessary and sufficient condition for the equation $\Psi_{a b}(w)=\Psi_{b a}(w)$ is that both of the equations $|w|_{a}=|w|_{b}$ and $|w|_{a b}=|w|_{b a}$ hold. According to Theorem 3, no unambiguous word satisfies these equations.

Another simple observation is the following

Lemma 3. The equation

$$
\Psi_{a b}(w)=\Psi_{b a}(w), w \in\{a, b\}^{*}
$$

implies that $|w|_{a}=|w|_{b}$ is even.

Proof. As seen above, we must have $|w|_{a}=|w|_{b}$. This number being odd would contradict the equation $\left(|w|_{a}\right) \cdot\left(|w|_{b}\right)=|w|_{a b}+|w|_{b a}$.

Consider now permutations $p$ of the (ordered) set $\left\{a_{1}, \ldots, a_{k}\right\}$. We apply such permutations to words letterwise. For instance, if $p$ is the circular permutation $(a b c)$, then $p(a c a b c b)=b a b c a c$.

Definition 5. A permutation $p$ of the set $\left\{a_{1}, \ldots, a_{k}\right\}, k \geq 2$, is Parikh-friendly if there is a word $w$, containing all of the letters $a_{1}, \ldots, a_{k}$ and referred to as a witness, such that

$$
\Psi_{a_{1} \cdots a_{k}}(w)=\Psi_{p\left(a_{1} \cdots a_{k}\right)}(w)
$$


It is necessary to assume that the witness word $w$ contains all the letters because, otherwise, every permutation is trivially Parikh-friendly.

For instance, consider the alphabet $\{a, b, c, d, e\}$. The circular permutation $p=(a b c d e)$ is Parikh-friendly. Choosing $w=a b c d e e d c b a$ we get

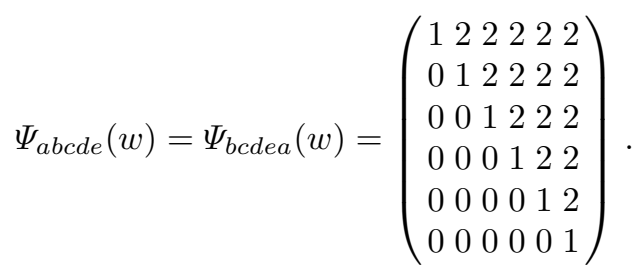

The example is a special case of the following result.

Theorem 6. For any $k \geq 2$, the circular permutation $p=\left(a_{1} a_{2} \cdots a_{k}\right)$ is Parikh-friendly.

Proof. The argument is as in the example. We choose $w=a_{1} a_{2} \cdots a_{k} a_{k} \cdots a_{2} a_{1}$ and observe that both $\Psi_{a_{1} a_{2} \cdots a_{k}}(w)$ and $\Psi_{a_{2} a_{3} \cdots a_{k} a_{1}}(w)$ assume as their value the Parikh matrix, where every entry above the main diagonal equals 2 .

The proof of Theorem 6 does not work for arbitrary circular permutations.

It is well-known that every permutation can be represented as a product of transpositions. The product of transpositions (in any order), where no two transpositions have a common element, is Parikh-friendly. Consider the alphabet $\{a, b, c, d, e, f, g\}$ and the permutation $p=(a e)(b c)(d f)$. (Thus, $p(g)=g$.) Choose $w=a e e a b c c b d f f d g g$. Then

$$
\Psi_{\text {abcdef } g}(w)=\Psi_{p(\text { abcdef })}(w)=\left(\begin{array}{llllllll}
1 & 2 & 4 & 4 & 8 & 0 & 0 & 0 \\
0 & 1 & 2 & 2 & 4 & 0 & 0 & 0 \\
0 & 0 & 1 & 2 & 4 & 0 & 0 & 0 \\
0 & 0 & 0 & 1 & 2 & 0 & 0 & 0 \\
0 & 0 & 0 & 0 & 1 & 2 & 4 & 8 \\
0 & 0 & 0 & 0 & 0 & 1 & 2 & 4 \\
0 & 0 & 0 & 0 & 0 & 0 & 1 & 2 \\
0 & 0 & 0 & 0 & 0 & 0 & 0 & 1
\end{array}\right) .
$$

In the witness word $w$ the order of the transpositions, as well as the fixed letter $g$, is irrelevant. It is also irrelevant whether we have one or two copies of $g$.

The example is a special case of the following result. However, it is an open problem to characterize Parikh-friendly permutations explicitly.

Theorem 7. Every product of transpositions, where no two transpositions have a common element, is Parikh-friendly. 
Proof. The construction of the witness follows the lines of the example. Consider the permutation $p$ of the alphabet $\left\{a_{1}, \ldots, a_{k}\right\}$, defined by

$$
p=\prod_{i=1}^{t}\left(b_{i}, c_{i}\right) \prod_{j=1}^{u}\left(d_{j}\right), 2 t+u=k .
$$

Here, the $b$ 's, $c$ 's and $d$ 's are all different and exhaust the alphabet $\left\{a_{1}, \ldots, a_{k}\right\}$ in some order. Define now

$$
w=\prod_{i=1}^{t} b_{i} c_{i}^{2} b_{i} \prod_{j=1}^{u} d_{j}
$$

It is easy to verify that

$$
\Psi_{a_{1} \cdots a_{k}}(w)=\Psi_{p\left(a_{1} \cdots a_{k}\right)}(w)
$$

\section{Degree of $M$-Ambiguity}

We conclude this paper with some remarks concerning a very natural notion not at all studied by now.

A context-free language can be of any degree of ambiguity, including infinity. For unambiguous languages the degree of ambiguity is one. Analogous notions can be defined for $M$-ambiguous words and Parikh matrices.

Definition 6. The degree of ambiguity $d(M)$ of a Parikh matrix $M$ is the number of words $w$ such that $\Psi(w)=M$. If $u$ is one of such words, then the degree of $M$-ambiguity of $u$ is defined to be $d(M)$.

$M$-unambiguous words are understood to have the degree of $M$-ambiguity 1 . Contrary to the situation in language theory, no word can be of infinite degree of $M$-ambiguity. This follows because all words $w$ satisfying the condition $\Psi(w)=$ $M$ have to be of same length.

However, the degree of $M$-ambiguity of a word can be exponential in terms of the length of the word. Consider the alphabet $\{a, b\}$ and the word

$$
w(n)=a^{n} b^{n} b^{n} a^{n}, \quad n \geq 1 .
$$

Clearly,

$$
\Psi(w(n))=\left(\begin{array}{ccc}
1 & 2 n & 2 n^{2} \\
0 & 1 & 2 n \\
0 & 0 & 1
\end{array}\right)
$$

The same matrix results whenever one replaces in $w(n)$ a factor $a b$ with the factor $b a$ and simultaneously replaces a factor $b a$ with the factor $a b$. The words obtainable in this fashion can be characterized as follows. Consider the $2 n$ occurrences of $a$ in a word $u$ resulting from $w(n)$ after a sequence of such 
simultaneous replacements. Denote by $x_{i}, 1 \leq i \leq 2 n$, the number of $b$ 's occurring to the right if the $i$ th occurrence of $a$. Thus, for $w(n)$ we have

$$
x_{1}=\ldots x_{n}=2 n, \quad x_{n+1}=\ldots x_{2 n}=0 .
$$

The words $M$-equivalent to $w(n)$ are determined by the following system of equations and inequalities:

$$
\sum_{i=1}^{2 n} x_{i}=2 n^{2}, 2 n \geq x_{i} \geq 0, x_{i} \geq x_{i+1} .
$$

Here in the first (resp. second) system of inequalities $i$ ranges from 1 to $2 n$ (resp. $2 n-1)$. We will see that the number of solutions is exponential in $n$.

For instance, take $n=4$. The solution

$$
x_{1}=7, x_{2}=x_{3}=5, x_{4}=4, x_{5}=x_{6}=x_{7}=3, x_{8}=2
$$

defines the word babbaababaaababb.

We now prove that the number of words with the Parikh matrix $\Psi(w(n))$ is exponential in $n$.

Lemma 4. There is no upper bound polynomial in $n$ for the number of words M-equivalent with $w(n)$.

Proof. Clearly,

$$
0 \leq|w|_{a b} \leq 4 n^{2}
$$

for words $w$ such that $|w|_{a}=|w|_{b}=2 n, n \geq 1$. We denote by $N(x), 0 \leq x \leq 4 n^{2}$, the number of words $w$ such that

$$
|w|_{a}=|w|_{b}=2 n,|w|_{a b}=x .
$$

By definition, $N\left(2 n^{2}\right)$ equals the number of words $M$-equivalent with $w(n)$. On the other hand, $N(0)=N\left(4 n^{2}\right)=1$, and $N(x)$ increases for $0 \leq x \leq 2 n^{2}-1$, and decreases for other values of $x$. The number of all words $w$ with $|w|_{a}=|w|_{b}=2$ equals the binomial coefficient $\left(\begin{array}{l}4 n \\ 2 n\end{array}\right)$, because these words are obtained by plotting $2 n$ occurrences of $b$ into $4 n$ positions. Consequently,

$$
\begin{aligned}
& N\left(2 n^{2}\right) \geq\left(\begin{array}{l}
4 n \\
2 n
\end{array}\right) /\left(4 n^{2}+1\right)=(4 n) ! /(2 n) ! \cdot(2 n) ! \cdot\left(4 n^{2}+1\right) \\
& =(4 n) \cdots(2 n+1) /(2 n) \cdots 1 \cdot\left(4 n^{2}+1\right)>2^{2 n} /\left(4 n^{2}+1\right),
\end{aligned}
$$

which proves the lemma.

A straightforward way of obtaining words whose degree of $M$-ambiguity is exponential in terms of word length is to consider the alphabet $\{a, b, c\}$. Then all words $w$ satisfying, for a given $n$,

$$
|w|_{a}=|w|_{c}=n,|w|_{b}=0
$$


have the Parikh matrix

$$
\left(\begin{array}{llll}
1 & n & 0 & 0 \\
0 & 1 & 0 & 0 \\
0 & 0 & 1 & n \\
0 & 0 & 0 & 1
\end{array}\right) .
$$

The number of these words equals the binomial coefficient $\left(\begin{array}{c}2 n \\ n\end{array}\right) \geq 2^{n}$. Thus, we obtain at least $2^{\sqrt{m}} M$-equivalent words of length $m$.

We have established the following result.

Theorem 8. Let $\Sigma$ be an alphabet with cardinality at least 2. There is no polynomial $P(n)$ such that, for all $n \geq 1$, the degree of $M$-ambiguity of an arbitrary word of length $n$ over $\Sigma$ is less than $P(n)$.

\section{Conclusion}

Many problems remain open concerning M-ambiguity and subword indicators. Tex has introduced the notion of strong $M$-equivalence and the resulting notion of strong $M$-ambiguity, [17]. By definition, two words are strongly $M$-equivalent if they are $M$-equivalent for all orderings of the alphabet. For instance, the word babcbabcbabcbab is strongly $M$-ambiguous. Tex has also initiated, [16], detailed studies about Parikh rewriting systems. We hope to return to these matters in another paper.

Acknowledgements. The author would like to thank the referee for careful reading of the paper and the editors for technical help concerning the final version of the paper.

\section{Dedication}

This article is dedicated to Juraj Hromkovič on the occasion of his 60th birthday. The article is not connected to any of Juraj's work. However, I still consider it suitable for this volume, in view of Juraj's most diverse and variegated work. I got to know Juraj already in the 80's in Bratislava, and he visited Turku and participated in the Tampere ICALP in 1988. After that he paid numerous visits to Turku and formed close contacts to some members of my research group and to researchers close to my group. I am very grateful for Juraj's participation in some festival conferences organized in Turku. In my view, Juraj's scientific work is deep, sometimes seminal. I admire his ability to combine science with innovative ideas about teaching. I think that some of his new ideas, such as the ones concerning the $P$ versus $N P$ problem, are not yet fully recognized. Dear Juraj, I wish you many more successful years in science and teaching, as well as health and happiness in life. 


\section{References}

1. Ding, C., Salomaa, A.: On some problems of Mateescu concerning subword occurrences. Fundam. Inform. 73, 65-79 (2006)

2. Eilenberg, S.: Automata, Languages and Machines, vol. B. Academic Press, New York (1976)

3. Mateescu, A., Salomaa, A., Salomaa, K., Yu, S.: A sharpening of the Parikh mapping. Theor. Inform. Appl. 35, 551-564 (2001)

4. Mateescu, A., Salomaa, A., Yu, S.: Subword histories and Parikh matrices. J. Comput. Syst. Sci. 68, 1-21 (2004)

5. Mateescu, A., Salomaa, A.: Matrix indicators for subword occurrences and ambiguity. Int. J. Found. Comput. Sci. 15, 277-292 (2004)

6. Parikh, R.J.: On context-free languages. J. Assoc. Comput. Mach. 13, 570-581 (1966)

7. Salomaa, A.: On the injectivity of Parikh matrix mappings. Fundam. Inform. 64, 391-404 (2005)

8. Salomaa, A.: Connections between subwords and certain matrix mappings. Theor. Comput. Sci. 340, 188-203 (2005)

9. Salomaa, A.: Independence of certain quantities indicating subword occurrences. Theor. Comput. Sci. 362, 222-231 (2006)

10. Salomaa, A.: Criteria for the matrix equivalence of words. Theor. Comput. Sci. 411, 1818-1827 (2010)

11. Salomaa, A.: Subword balance, position indices and power sums. J. Comput. Syst. Sci. 76, 861-871 (2010)

12. Salomaa, A.: On a Cauchy-type inequality for subword occurrences. Analele Universitate Bucuresti 62(2), 101-112 (2015)

13. Şerbănuţă, T.-F.: Extending Parikh matrices. Theor. Comput. Sci. 310, 233-246 (2004)

14. Şerbănuţă, V.G., Şerbănuţă, T.F.: Injectivity of the Parikh matrix mappings revisited. Fundam. Inform. 73, 265-283 (2006)

15. Şerbănuţă, V.G.: On Parikh matrices, ambiguity and prints. Int. J. Found. Comput. Sci. 20, 151-165 (2009)

16. Tex, W.C.: Parikh matrices and Parikh rewriting systems. Fundam. Inform. 146, 305-320 (2016)

17. Tex, W.C.: On $M$-ambiguity for Parikh matrices. In: Asian Mathematical Conference, AMC 2016 (2016)

18. Teh, W.C., Atanasiu, A.: On a conjecture about Parikh matrices. Theor. Comput. Sci. 628, 30-39 (2016)

19. Tex, W.C., Atanasiu, A., Poovanandran, G.: On strongly $M$-ambiguous prints and Şerbănuţă's conjecture for Parikh matrices (2017, submitted) 